\title{
Tecnura
}

http://revistas.udistrital.edu.co/ojs/index.php/Tecnura/issue/view/640

DOI: http://dx.doi.org/10.14483/udistrital.jour.tecnura.2014.SE1.a19

ReFLEXIÓN

\section{La formación lingüística en la educación superior}

\section{Linguistic education in higher education}

\author{
Nevis Balanta Castilla*, David Navarro Mejía**
}

Citation / Para citar este artículo: Navarro Mejía, D., \& Balanta Castilla, N. (2014). La formación lingüística en la educación superior. Revista Tecnura, Edición especial, 255-262.

Fecha de recepción: 29 de noviembre de 2013 / Fecha de aceptación: 11 de julio de 2014

\section{Resumen}

En este artículo se hace un recorrido por los enfoques y el estado de la educación lingüística en la educación superior en Colombia, pero al mismo tiempo su situación se articula a cómo se le ha dispuesto en la educación básica y media, en el entendido que muchas de las carencias de la educación lingüística en las universidades no tienen explicación razonable sin una aproximación de lo que sucede en esos otros niveles del sistema educativo. Por eso se acude también al registro de algunas cifras de las pruebas PISA que indican las carencias evidentes en el aprendizaje del lenguaje. Se presenta, además, un recuento breve de lo que de algún modo es el mapa de los micro-currículos que se ofrecen en este campo en las universidades, y se ponen en contexto las competencias que en el área de lenguaje se evalúan en las pruebas Saber Pro. Finalmente, se propone una ruta de lo que tendría que revisarse para mejorar la educación lingüística en la educación superior en Colombia.

Palabras clave: educación lingüística, competencias en lenguaje, enfoques de la educación lingüística, micro-currículo, alfabetización académica, escritura a través del currículo.

\begin{abstract}
This article presents an overview of the approaches and the state of linguistic education in higher education in Colombia, but at the same time, its situation is articulated to how it has been arranged in primary and secondary education, based on the idea that most of the scarcities in linguistic education at universities do not have a reasonable explanation without an approach to what is occurring in those other levels of the educational system. Thus, recurring to the register of some statistics from PISA tests indicate the evident scarcities in language learning. Moreover, a brief outlook of what is some way the map of the micro-curriculum offered in this field at universities is presented, and a contextualization of the competences that in the area of language are evaluated in Saber Pro tests. Finally, there is a proposal for a path of what should be reviewed to improve the linguistic education in higher education in Colombia.
\end{abstract}

Keywords: Linguistic education, language competences, linguistic education approaches, micro-curriculum, academic literacy, writing across the curriculum.

\footnotetext{
* Licenciada en lingüística y literatura, magíster en investigación social interdisciplinaria. Docente, Universidad Distrital Francisco José de Caldas. Bogotá, Colombia. Contacto: nbalantac@udistrital.edu.co

** Licenciado en lingüística y literatura; magíster en investigación social interdisciplinaria; doctor en ciencias de la información. Docente, Universidad Distrital Francisco José de Caldas. Bogotá, Colombia. Contacto: sec-tecnologica@udistrital.edu.co
} 


\section{PRESENTACIÓN}

Cada vez es más reiterativa la preocupación por el bajo desempeño y rendimiento académicos de los estudiantes de educación superior respecto de sus competencias en el uso del lenguaje. Se entiende que si bien estas hacen parte de las Ilamadas competencias generales -en apariencia nada importantes para la vida profesional-, de todos modos al tener noticias por las pruebas PISA que evalúan la capacidad lingüística de los estudiantes y su incidencia en la lectura y la escritura, no sale bien librado nuestro sistema educativo, ni el país como el más aventajado en el uso de nuestra lengua. Este aspecto es tanto más sensible por cuanto las carencias en el uso del lenguaje tienen también implicaciones directas en el aprendizaje de las ciencias y otros campos que son clave para el desarrollo humano. De aquí, el sentido que cobra repensar cómo se está haciendo la educación lingüística en la educación superior.

\section{ENFOQUE DE ENSEÑANZA DEL LENGUAJE EN LA EDUCACIÓN BÁSICA Y MEDIA}

En 1998 el MEN publicó lo que Ilamó los lineamientos para la enseñanza y construcción de los currículos del lenguaje. Ese enfoque del MEN hacía énfasis en la semántica comunicativa. Dicha visión se concretó en un documento que el MEN construyo en 1998 y con arreglo al cual, la enseñanza del lenguaje buscaba desarrollar no solo competencias lingüísticas sino también competencias comunicativas que se creía eran más apropiadas y abarcadoras y estaban más a tono con los nuevos estudios del lenguaje que señalaban la importancia de su dimensión social y cultural.

Se criticaba en aquel momento el énfasis demasiado normativo de la enseñanza de la lengua porque presumiblemente los "currículos en el campo de lenguaje estaban marcados por un gramaticalismo y memorización de las normativas lingüísticas" (1998, p. 46). Gran parte de la transformación que hubo entonces se hizo como impugnación a ese enfoque y se asumió, bajo la concepción de dos autores y visiones: la una, propugnada por Dell Hymes, quien planteó la noción de competencia comunicativa como aquella que trasciende el uso del lenguaje en actos comunicativos particulares que se dan en el entorno socio-cultural (ibid.), y la otra, la del maestro colombiano Luis Ángel Baena, que sostenía que la función central del lenguaje era la significación, además de la comunicación. (p. 47).

Se entiende que esa perspectiva supuso una visión interdisciplinaria, que integraba la semiótica, la sociología y la historia como disciplinas cuyos saberes le reportaban explicaciones valiosas a los fenómenos del lenguaje y ampliaba su repertorio de códigos con el que se produce la comunicación.

Desde luego, aquella concepción del lenguaje sigue teniendo una vigencia notable en la actualidad, pero no contamos con un diagnóstico detallado de cuáles han sido sus resultados. Un indicador de eso concebimos que son las pruebas PISA que evalúan conocimientos y competencias en lectura. Sobre este particular, estudios recientes han señalado las carencias que siguen teniendo nuestros estudiantes en el área de lenguaje.

Según el texto Separados y desiguales datos del año 2012 indican que Colombia, entre 65 países que participaron de la prueba, ocupó el puesto 57 en lenguaje, lo cual se traduce en un bajo desempeño con respecto a los países de la OCDE. De acuerdo con esos resultados, Colombia en el área de lectura obtuvo en promedio 403 puntos, por detrás de países de la región como Chile (441), Costa Rica (441), México (424), Uruguay (411) y Brasil (410). Solo superó a Argentina (394) y Perú (384) (pp. 29-30).

Por otra parte, el documento del MEN Estándares básicos de competencias establece los siguientes factores por trabajar en la educación lingüística: producción textual, comprensión e interpretación textual, literatura, medios de comunicación y otros sistemas simbólicos y ética de la comunicación. Define a su vez unos propósitos (enunciado identificador) y unos subprocesos que se deben cumplir.

De lo que allí se plantea, lo relevante es que los micro-currículos y los manuales escolares en 
educación básica y media disponen la producción textual de esquemas y resúmenes; la lectura de fábulas, cuentos y leyendas, la identificación de formatos de los medios, entre otros aspectos en el nivel de primaria; la producción textual de mapas mentales, reseñas y ensayo, la identificación de géneros periodísticos de los medios y entre programas de la televisión, y de los géneros literarios, así como obras literarias latinoamericanas, acercándose a ellas desde una posición crítica y argumentada. Se dispone también defender las posiciones contrarias y el respeto por la diversidad de nuestra cultura.

Este repertorio de temas y propósitos para la educación básica y media tiene como novedad el interés por acercarse a otros sistemas de lenguaje, pero siempre queda la duda de si la organización y cantidad de información que se prescribe es la adecuada para desarrollar las competencias de los estudiantes en lenguaje. En esa organización curricular se dispone, además, un enfoque que ahora añade lo discursivo como un eje central de los conocimientos y competencias que debe aprender el estudiante.

Es pertinente, por tanto, revisar qué tanto viene influyendo en las carencias que se señalan en las competencias lingüísticas de los estudiantes un sinnúmero de estándares que se suponen básicos, pero que quizá han trascendido ese límite y serían más propios de grados superiores a la educación media y básica. Pero de aquí también la justificación de por qué se ha abordado lo que en materia de lenguaje se ha dispuesto en los niveles que preceden a la educación superior.

\section{BREVE DIAGNÓSTICO DEL ESTADO GENERAL DE LAS DENOMINACIONES DE LOS MICRO-CURRÍCULOS Y LO QUE SE ENSEÑA EN LENGUAJE EN LA EDUCACIÓN SUPERIOR}

Según una consulta que se realizó desde los portales y contactos directos con las universidades en el año 2011, estas conciben espacios académicos cuyas denominaciones son distintas, pero su contenido apunta a la enseñanza de saberes básicos y avanzados de la lengua con el fin de desarroIlar las competencias comunicativas de los estudiantes. Las denominaciones encontradas fueron: Taller de lenguaje, Producción y comprensión de textos, Habilidades comunicativas, Técnicas de la comunicación oral y escrita, Comunicación oral y escrita, Español, Competencias comunicativas, Lecto-escritura, Gramática, Comunicación y lenguaje, Taller de comprensión y producción de textos, Taller de lectura, Taller de escritura, Retórica y expresión, Propedéutica de textos, Escritura de ensayos de opinión, Análisis de textos, Artes narrativas, Argumentación, Análisis de argumentos, etc.

Aquella investigación se hizo teniendo como punto de partida la información que se consiguió a través de la red REDLES, constituida por universidades de Bogotá que trabajan en los procesos de lectura y escritura de sus estudiantes. Hasta 2011 dicha red la constituían treinta universidades.

Se analizó en esa investigación la estructura de los micro-currículos de las universidades seleccionadas y se estableció una ficha de evaluación que agrupó su contenido en dos campos: un componente pedagógico (que incluía el propósito general, el enfoque pedagógico y la justificación) y un componente didáctico (que incluía estrategias didácticas, objetivos generales y específicos, estructuración del programa y componentes, formas de evaluación y recursos).

Las conclusiones preliminares de ese estudio arrojó lo siguiente:

- Que los micro-currículos más que formalizarlo, debe atender a que cada elemento de su constitución responda en verdad a los criterios formales que las universidades han definido para que sus contenidos, metodología, estrategias y recursos sean coherentes con esas condiciones institucionales.

- Los contenidos temáticos del micro-currículo deben ser ordenados de acuerdo con los propósitos: leer y escribir mejor, aspectos formales de la lengua española, géneros discursivos de la academia, o la suma coherente de todas estas partes. 
Esa decisión no parece clara en los actuales contenidos de los micro-currículos de las asignaturas relacionadas con la lengua española.

- Los textos escolares deben ser diseñados en un lenguaje claro, es decir, deben ser ejemplo de lo que pretenden que los estudiantes aprendan; visualmente atractivos; conceptual y teóricamente pertinentes y moderadamente realistas en sus propósitos.

- Si la lengua castellana no solo sirve para comunicarnos, sino también para acceder a los saberes y conocimientos que nos han legado la ciencia y la cultura, los géneros discursivos en los que se despliegan esos saberes deben tener prioridad en la producción de textos que elaboran los estudiantes, pero eso exige una renovada importancia de las materias relacionadas con el lenguaje en los currículos de las carreras universitarias, en virtud de que los espacios y la intensidad horaria con que se dispone hoy día en las universidades no está acorde con las exigencias que hoy impone la enseñanza de la lengua española o sus materias similares o afines en los programas universitarios.

\section{VISIÓN DE LA ENSEÑANZA EN LENGUAJE EN LA EDUCACIÓN SUPERIOR}

Ahora bien, cuando se dispone la enseñanza de nuestra lengua, no siempre existe la claridad sobre su papel en el marco de otros lenguajes que también están presentes en el universo de la comunicación. Por lo regular, su enseñanza supone que en un lugar del proceso educativo se debe abordar el significado de las imágenes y del lenguaje literario, cifrado en el uso de metáforas, hipérboles y otros tropos. Las imágenes suponen además, en su descripción y explicación, signos como los símbolos, los iconos y otros no verbales.

La enseñanza se centra pues, en la pluralidad de lenguajes. La lengua castellana se centra aún en la oración y se amplía a las partes y la estructura de un texto. No obstante, se ha subestimado el entrenamiento en la función que cumplen las palabras bajo el supuesto de su conocimiento previo, o de su carácter gramatical que no es muy apreciado. Por eso se ha orientado también la educación lingüística al estudio de géneros discursivos. Pero determinar sus estructuras textuales requiere conocer las unidades lingüísticas desde el punto de vista formal y eso se ve obstaculizado por cierta visión que desdeña la gramática de la lengua, o esconde detrás de su saber su evolución y transformación. Esa gramática, en cambio, se acepta sin críticas cuando se la enseña en lenguas extranjeras. Dicha visión supone además una connotación negativa de su significado.

Por eso es evidente que para determinar el currículo de la enseñanza en lenguaje, es clave lo que en otras culturas han definido en este campo. Y cómo la ubican dentro de las competencias que debe registrar un profesional. En este sentido, a la educación lingüística la han inscrito en lo que se llaman competencias generales, ámbito en cuyos límites se encontraría el lugar de ubicación del lenguaje en los programas que se ofertan en la educación superior, salvo la que educa para la enseñanza y profesión en dicho campo.

La American Association of Colleges and Schools, AACU 2007, por ejemplo, identifica para cualquier profesión lo que denomina competencias intelectuales y prácticas, para lo que incluye en ellas la comunicación oral y escrita (Toro, pp. 236-240). La importancia y creciente interés en estas competencias, según Toro, es que los desafíos laborales y humanos de los profesionales de hoy no están necesariamente en las capacidades profesionales, sino que "una buena parte se asocia a lo que llamamos competencias generales", más cuando estudios efectuados en muchas empresas de Estados Unidos reportan que los empleadores adjudican mucha importancia a muchas de las competencias generales que hasta hace poco se desdeñaban. (p. 243). Explica también este fenómeno el hecho de que en años recientes una persona pueda tener durante su vida profesional muchos empleos y lo más probable es que en su recorrido haya una "mutación profesional" que hace que las competencias generales, en determinados 
momentos, sean las que dan una ventaja para mantenerse activo en su campo, o para migrar a otro campo profesional.

Por otra parte, un elemento adicional para el diseño de la educación lingüística es que Colombia no puede pretender un diseño curricular que ignore la procedencia social de los estudiantes, pues la evidente desigualdad en la educación constituye uno de los factores que obstaculizan la oferta de una educación lingüística de calidad. Quizá sea necesario, por tanto, una educación diferencial en lenguaje que promueva mayor igualdad en el aprendizaje de los estudiantes de educación superior.

\section{¿QUÉ EDUCACIÓN LINGÜÍSTICA PARA LA EDUCACIÓN SUPERIOR?}

En este contexto el interrogante resulta apenas oportuno. Entre las respuestas probables, habría que decir que en los últimos años se ha despertado una preferencia en la enseñanza de la lengua por la construcción discursiva de la misma otorgando un papel especial a su nivel pragmático. En este sentido, vale señalar que se ha determinado la enseñanza de algunos géneros que se creen válidos en este ciclo, como el resumen, el informe $y$, sobre todo, el ensayo. Desafortunadamente la experiencia muestra que su aprendizaje está atravesado por una serie de factores de carácter cognitivo y social que no siempre tienen la atención debida en los currículos que se deciden para enseñar.

Por otra parte, las Pruebas Saber Pro tienen una orientación que no siempre es coherente con lo que se enseña en la educación superior, dado el sentido de algunos de los micro-currículos. El caso es que en las mencionadas pruebas las competencias comunicativas que se evalúan, apuntan a:

- Identificar elementos locales del texto.

- Identificar las partes que constituyen el todo de un texto.

- Identificar las estrategias discursivas de un texto.
Todo ello, según el propósito de las pruebas, debe arrojar la realización de una lectura crítica de los textos.

En el caso de la producción textual, en las pruebas "En los escritos se evalúa la manera como se estructuran, organizan y comunican las ideas; en particular, se tienen en cuenta la organización en la exposición de las ideas, la conexión entre los distintos tópicos, la selección del lenguaje más apropiado, el dominio de las reglas de la expresión escrita y la claridad con que se perfila la relación con el lector. Se analiza panorámicamente si las estrategias del autor fueron adecuadas para su propósito, independientemente de si el texto tiene un formato determinado".

Vale decir entonces que las pruebas centran la evaluación en la estructura y organización del texto, sin atender lo que Ilamamos los géneros discursivos, aspecto que quizá sea una limitación, si se tiene en cuenta que el repertorio de temas que se abordan en los micro-currículos de las universidades están inclinados a la enseñanza desde una perspectiva discursiva como recordábamos atrás, requisito que se considera indispensable para el desempeño profesional.

En cualquier caso, un repertorio de esos micro-currículos registra estudios en lo siguiente: gramática, argumentación, géneros como el resumen, informe, la reseña y el ensayo; retórica, narrativa, habilidades comunicativas y análisis de textos, entre otros. Es decir, hay en general los contenidos que se suponen adecuados para la educación lingüística en educación superior. Pero es también evidente que su diseño y organización curricular están atravesados por una mezcla de los enfoques estructuralista y comunicativo de enseñanza de la lengua. Si bien de modo formal se prescriben enfoques que parecen privilegiar el uso concreto, real y experiencial de la lengua escrita y oral, también lo es que el diseño de muchos micro-curriculos no revela con claridad esa apuesta, sino que más bien representa una mixtura de los enfoques mencionados, más el énfasis que se ha puesto en los discursos del lenguaje, en virtud del 
aporte de los últimos años en campos como la lingüística textual, el análisis del discurso, la semiótica y otros afines.

Pero más allá de esas prescripciones de la enseñanza del lenguaje, las dificultades que se reportan de los estudiantes para aprender y ser más competitivos en este campo parecen estribar en otros ámbitos que de pronto no han ocupado la atención de las universidades. Al respecto, son pertinentes los estudios que se han realizado para determinar problemas de enseñanza con los estudiantes universitarios. Algunos de esos estudios, por ejemplo, señalan que en el trabajo de corrección y evaluación de los textos "en todos los casos, el profesor no hace consciente que el texto que finalmente evalúa no es el del alumno, sino el que resulta de su lectura (y en gran parte reescritura) del texto del alumno" (Desinano, 2009, p. 50).

Para esta autora, según su planteamiento, lo que usualmente en la enseñanza se asume como error o fallo de los alumnos no es más que el desconocimiento de cómo se inscriben ellos como sujeto del lenguaje, lo cual marca una diferencia en la manera de concebir y entender el uso del lenguaje. Y remarca, como se ve en muchos en la actualidad, que se hace demasiado énfasis en el estudio del sistema de la lengua, o del lenguaje, cuando es fundamental la interacción de donde se deriva su uso y realización.

En esta misma dirección apuntan los estudios que intentan destacar el ámbito de uso del lenguaje desde la experiencia escritural de los propios docentes, como muestra Finocchio en la experiencia argentina de los profesores (2009) quien, al reflexionar sobre la escritura escolar, impugna la crítica sobre las carencias que se atribuyen a los estudiantes, a partir de poner en escena la escritura de los propios docentes en su condición no ya de enseñantes, sino de escritores, y establece que es importante esa escritura para develar las propias dificultades de los estudiantes.

En la perspectiva de crear un mapa más adecuado para la enseñanza del lenguaje en la educación superior, vale registrar también los usos de los libros de texto, pues se presenta una especie de interés renovado con la edición de libros focalizados para este segmento de la educación, lo cual revela que se ha entendido que su enseñanza también exige textos de referencia para este nivel. A modo de muestra, cabe mencionar algunos que han circulado en los últimos años por los ámbitos universitarios, como Escribir, leer y aprender en la Universidad, de Paula Carlino, Cómo se escribe, de María Teresa Serafini y La Cocina de la escritura y Afilar el lapicero, de Daniel Cassany, textos que se cree han sido referencia para la enseñanza en la educación superior y cuya selección ya marca un indicio del enfoque cómo se está concibiendo la enseñanza del lenguaje.

Estos textos, independientemente de su pertinencia, hay que notar que proponen una enseñanza del lenguaje que se sustenta en el concepto de alfabetización académica, que se entiende como el campo de saberes y prácticas que se agrupan en las actividades encaminadas al aprendizaje en la universidad, a partir de aprender la producción y análisis de textos y las nociones y estrategias que se requieren para acceder a la cultura discursiva de las disciplinas y profesiones en las que se educan los estudiantes universitarios. Y proceso mediante el cual se adquiere carta de ciudadanía intelectual en una comunidad científica y profesional (Flórez y Gutiérrez, 2011. p. 17).

A nuestro modo de ver, sin embargo, la alfabetización académica se promueve más del deber ser que de lo que es la escritura en acción y en concreto, con todos sus fallos, de los estudiantes. Es decir, prefigura más un modo de ver la práctica de la escritura, que lo que es realmente el universo de la escritura para el estudiante cuando se le requiere el uso del lenguaje para esta actividad. Por eso es pertinente señalar que la defensa y promoción del concepto de alfabetización o de escritura académica, como una suerte de diferenciar la enseñanza del lenguaje en la educación superior, debe someterse a evaluación y examen previos, como una opción de enfoque en la educación superior. 
Es claro que Carlino y Cassany se han apoyado en este enfoque de escritura en la universidad, o escritura en las disciplinas y profesiones en las que se forman los estudiantes de educación superior, en experiencias, programas y enfoques que se iniciaron desde la década de 1980 en Estados Unidos, Europa y Australia, casos particulares de la Universidad de Pensylvania, la Universidad de Princeton, la Universidad de Columbia, la Universidad de Brown y la Universidad Pompeu de Fabra. Habría que agregar que dicho enfoque se complementa y apoya en el concepto de Escritura a través de currículo (por sus iniciales en inglés: Writing Across the Curriculum, WAC), cuyo surgimiento e impulso se hizo desde las Universidades de California y de Berkeley, concepto que a su vez tiene su antecedente en otro acuñado a finales de la década de 1960 en Gran Bretaña: el lenguaje a través del curriculum (LAC). Es una visión desde la cual la enseñanza del lenguaje no se asume como enseñar contenidos, sino como una práctica que se ejerce y enseña desde todas las disciplinas y saberes, incluidos por supuesto, los de la propia lengua en que se escribe.

Por otra parte, en Colombia la Universidad Nacional de Colombia en 2001 creó e impulsó el programa UNESCRIBE que pretendía situar la escritura como centro de sus preocupaciones académicas en el interés de habilitar a los estudiantes como practicantes competentes de las comunidades discursivas de las disciplinas en las que se educan. Y en 2006 se creó la red REDLEES que agrupa varias instituciones de educación superior que promueven la lectura y escritura con un énfasis renovado, amén de que universidades como la Javeriana, la Sergio Arboleda y la Institución Universitaria Iberoamericana han promovido desde instancias distintas programas y proyectos que buscan situar la enseñanza del lenguaje en el centro de sus preocupaciones académicas. Es decir, hay un nuevo contexto que es preciso evaluar para reorientar la educación lingüística en la educación superior colombiana.

Cabe, de igual modo, la pertinencia del debate sobre el uso de las Ilamadas nuevas tecnologías asociadas con la información y las comunicaciones, que han supuesto la construcción de una lengua y un lenguaje que acude a códigos propios que según la visión que se tenga, dificultan a veces la enseñanza del español, pero también la pueden favorecer. Y no es desdeñable, tampoco, el interés por aprender una lengua extranjera que ha supuesto quizá, paradójicamente, un desinterés y desmotivación por el dominio y conocimiento competente de la lengua materna.

Y también sería válida una revisión de las estrategias didácticas y pedagógicas, más cuando el uso de internet y las nuevas tecnologías vienen revaluando el rol de los docentes en la educación superior y en todo el sistema. Por eso, no es irrelevante revisar, con detalle, qué tanto conocimiento se debe ofrecer en los micro-currículos, pues siempre se ha tenido la tentación de incorporar el mayor cúmulo de saberes, cuando es evidente que el fortalecimiento de competencias en el uso del lenguaje no debe terminar en esos espacios académicos.

Dicho lo anterior, se considera que la educación lingüística en educación superior debe revisar sus currículos para poner el acento en los siguientes tópicos:

- Énfasis en el enfoque discursivo de la lengua.

- Evaluar y adecuar de manera diferencial, la pertinencia de incorporar el enfoque que se deriva del concepto Escritura a través del Currículo.

- Revalidación de las estrategias didácticas en una construcción permanente.

- Revisión de los currículos actuales de los programas de lengua castellana y humanidades.

- Disponer una estrategia para minimizar las dificultades de aprendizaje del lenguaje con una organización más lógica y pertinente de los micro-currículos.

- Regresar a los programas de filología española y lingüística como campos de saber profesional específico que atraviesan la educación lingüística en la educación superior. 
Desde luego, no pretendemos que el estado y las perspectivas que ofrecemos en la educación lingüística que analizamos constituyan el cuadro completo de su examen y situación actual. Pero aspiramos que avive nuevamente el interés por la enseñanza en este campo para contribuir a mejorar la calidad de la educación superior.

\section{FINANCIAMIENTO}

\section{Universidad Distrital Francisco José de Caldas}

\section{REFERENCIAS}

AA.VV. Lengua castellana. Lineamientos curricularesáreas obligatorias y fundamentales. Bogotá, MEN.

Desinano, Norma B. (2009). Los alumnos universitarios y la escritura académica. Análisis de un problema. Rosario: Homo Sapiens.

Finocchio, Ana María (2009). Conquistar la escritura. Saberes y prácticas escolares. Buenos Aires: Paidós.
Flórez, Rita y Gutiérrez, Maureen (2011). Alfabetización académica: una propuesta para la formación de docentes universitarios. Bogotá: Universidad Nacional de Colombia.

García, Mauricio et al. (2013). Separados y desiguales. Educación y Clases Sociales en Colombia. Bogotá: DeJusticia.

Jurado, Fabio (1999). Investigación, escritura y educación. El lenguaje y la literatura en la transformación de la escuela. Bogotá: Universidad Nacional de Colombia / Plaza \& Janés.

Toro, José Rafael (2014). En: Educación Superior en Colombia. Roa Varelo, Alberto y Pacheco, Iván (eds.) Barranquilla, Universidad del Norte/ Center for International Higher Education. http://www.tecnar. edu.co/sites/default/files/pdfs/M\%C3\%B3dulo-COMUNICACION\%20ESCRITA.pdf. Recuperado 25 de julio de 2014. (http://www.mineducacion.gov. co/1621/articles-116042_archivo_pdf1.pdf: recuperado julio 25 de 2014. pp. 29-45). 